\title{
Fabrication, in vitro and in vivo studies of bilayer composite membrane for periodontal guided tissue regeneration
}

Journal of Biomaterials Applications 2019, Vol. 33(7) 967-978 (C) The Author(s) 2018 Article reuse guidelines: sagepub.com/journals-permissions DOI: 10.1 |77/08853282|88|4986 journals.sagepub.com/home/jba (SAGE

\author{
Saba Zahid', Abdul Samad Khan ${ }^{2}$ (D), Aqif Anwar Chaudhry', \\ Sarah Ghafoor ${ }^{3}$, Qurat UI Ain ${ }^{3}$, Ahtasham Raza ${ }^{4}$, \\ Muhammad Imran Rahim', Oliver Goerke', \\ Ihtesham Ur Rehman ${ }^{4}$ and Asma Tufail'
}

\begin{abstract}
Development of a guided occlusive biodegradable membrane with controlled morphology in order to restrict the ingrowth of epithelial cells is still a challenge in dental tissue engineering. A bilayer membrane with a non-porous upper layer (polyurethane) and porous lower layer (polycaprolactone and bioactive glass composite) with thermoelastic properties to sustain surgery treatment was developed by lyophilization. Morphology, porosity, and layers attachment were controlled by using the multi-solvent system. In vitro and in vivo biocompatibility, cell attachment, and cell proliferation were analyzed by immunohistochemistry and histology. The cell proliferation rate and cell attachment results showed good biocompatibility of both surfaces, though cell metabolic activity was better on the polycaprolactonebioactive glass surface. Furthermore, the cells were viable, adhered, and proliferated well on the lower porous bioactive surface, while non-porous polyurethane surface demonstrated low cell attachment, which was deliberately designed and a pre-requisite for guided tissue regeneration/guided bone regeneration membranes. In addition, in vivo studies performed in a rat model for six weeks revealed good compatibility of membranes. Histological analysis (staining with hematoxylin and eosin) indicated no signs of inflammation or accumulation of host immune cells. These results suggested that the fabricated biocompatible bilayer membrane has the potential for use in periodontal tissue regeneration.
\end{abstract}

\section{Keywords}

Guided tissue regeneration, bioactive glass, bilayer membrane, in vitro cytotoxicity, animal testing, immuno-staining

\section{Introduction}

Periodontitis, one of the most progressive oral disease, damages soft tissue and destroys tooth-supporting bone, often resulting in the loss of a tooth. ${ }^{1}$ The rate of periodontal breakdown has significantly been increased in the last few years. In recent years, guided tissue regeneration/guided bone regeneration (GTR/ GBR) approaches are being used extensively for the treatment of periodontitis. In both cases, an occlusive periodontal membrane that acts as a barrier to prevent down-growth of epithelial and connective tissues into the defect and enabling periodontal regeneration is used. ${ }^{2}$ The success of GTR/GBR membranes depends on achieving the repair of the large area through osteoconductivity, mechanical stability, and equilibrium

\footnotetext{
'Interdisciplinary Research Centre in Biomedical Materials, COMSATS University Islamabad, Lahore Campus, Lahore, Pakistan

${ }^{2}$ Department of Restorative Dental Sciences, College of Dentistry, Imam Abdulrahman Bin Faisal University, Dammam, Saudi Arabia

${ }^{3}$ Department of Oral Biology, University of Health Sciences Lahore, Khayaban-e-Jamia Punjab, Lahore, Pakistan

${ }^{4}$ Department of Material Science and Engineering, Kroto Research Institute, The University of Sheffield, Sheffield, United Kingdom ${ }^{5}$ Fachgebiet Keramische Werkstoffe / Chair of Advanced Ceramic Materials, Institut für Werkstoffwissenschaften und -technologien, Technische Universität Berlin, Hardenbergstr, Berlin, Germany

Corresponding author:

Asma Tufail, COMSATS University Islamabad, Lahore Campus, Lahore54000, Pakistan.

Email: drasmashah@cuilahore.edu.pk
} 
between the degradation rate of membranes and tissue regeneration in order to restrict epithelial cells ingrowth. Both natural (chitosan, collagen, etc.) and synthetic (polyurethane (PU), polycaprolactone (PCL), etc.) biopolymers are being used for the development of GTR membranes. ${ }^{3-5}$ PCL is a biodegradable polyester with good mechanical stability and bioactivity for endothelial cells. It degrades hydrolytically, and cells replace the degrading material by continuously infiltrating the matrix that produces collagen, elastin, and proteoglycans. ${ }^{6,7}$ Biodegradable PU is a synthetic polymer with good mechanical stability and tuneable microstructure due to flexible hard and soft segments. ${ }^{8}$ It has been used for soft tissue and bone regeneration in vivo and showed good biocompatibility and biodegradability for GTR membranes. The requirement of these biopolymers differs according to the nature of the application. ${ }^{9}$ The inclusion of bioceramics as filler in these polymers induces osteoconductive properties and accelerates the healing process. ${ }^{10}$ Among bioceramics, bioactive glasses (BGs) are known to have osteoproductive and osteostimulative properties. They have the ability to form an apatitelike layer at the interface in vivo by undergoing specific surface reactions, which leads to the development of a strong adherent bond with the host hard/soft tissues. Apatite layer stimulates osteoblasts (bone forming cells) and promotes new bone formation in situ. ${ }^{11}$ Furthermore, BGs have been used as filler with alginate and found to prevent the formation of fibrous tissue at the prosthesis-bone interface and showed in vivo new bone formation within 7 to 14 days. ${ }^{12}$ However, alginate possesses low intrinsic mechanical strength.

The ability of GTR membranes to seclude epithelial cells from the targeted area also depends on their morphology and porosity, ${ }^{13}$ which can be controlled by methodology and nature of the polymers. Freezedrying process is relatively a new technique to fabricate meso- and macroporous three-dimensional biomaterials. ${ }^{14}$ This process is based on phase separation mechanism (solid-liquid de-mixing). The shape and morphology of scaffolds can be influenced by modifying the concentration of polymers, using a mixture of solvents, or varying the lyophilization conditions such as cooling rate. ${ }^{15}$

In this work, bilayer GTR/GBR membranes with a non-porous lower layer (to exclude epithelial cells ingrowth) and porous upper layer (with the ability to regenerate bone) were fabricated using a freeze-drying method. In order to persuade biodegradability and fast bone and tissue healing properties, PCL and BG were chosen, while PU was selected due to its intrinsic mechanical stability for handling during surgery. The porosity of membranes was regulated by controlling the nature and concentration of polymers, solvents, and reaction conditions. A combination of solvents was used to control the sublimation rate and thus porosity. The in vitro as well as in vivo studies were performed to fully understand the biological properties of the membranes.

\section{Materials and methods}

Synthetic PU (PU, Z3A1, Biomer Technology Ltd., Runcorn, UK) and PCL (molecular weight: 80,000, Sigma Aldrich, UK) were used for developing the membrane. The solvents dioxane (Fischer Scientific, England) and dimethylformamide (DMF, Sigma Aldrich, UK) were of analytical grade, and no further purification was required before their use.

\section{Fabrication of bilayer GTR/GBR membranes}

BG was synthesized by a base-catalyzed sol-gel technique using calcium nitrate tetrahydrate $(\mathrm{Ca}$ $\left.\left(\mathrm{NO}_{3}\right)_{2} \cdot 4 \mathrm{H}_{2} \mathrm{O}\right)$, tetraethyl orthosilicate, and diammonium hydrogen phosphate $\left[\left(\mathrm{NH}_{4}\right)_{2} \mathrm{HPO}_{4}\right]$ as a source of calcium oxide $(\mathrm{CaO})$, silica $\left(\mathrm{SiO}_{2}\right)$, and phosphorous pentaoxide $\left(\mathrm{P}_{2} \mathrm{O}_{5}\right)$, respectively, following the procedure given previously. ${ }^{16}$ The synthesized BG was sintered at $680^{\circ} \mathrm{C}$. To prepare a bilayer membrane, the upper layer was composed of the pure PU and the lower layer consisted of analytical grade PCL with BG.

For the upper layer, $5 \mathrm{wt} . \%$ of the PU was added to a mixture of solvents (dioxane and DMF in a volume ratio of 4:1) at room temperature. The lower layer of the membrane was fabricated by dissolving 5 wt. $\%$ of the PCL in dioxane and stirred overnight to get a clear solution. Then, BG (40 wt. \%) was added to the prepared PCL solution. For fabrication of the bilayer membrane, the PCL and the BG mixture was added into a Petri-plate, cooled at $4^{\circ} \mathrm{C}$ for $2 \mathrm{~h}$ and frozen at $-20^{\circ} \mathrm{C}$. Then, a pre-cooled solution of the PU was poured over the PCL-BG frozen layer and kept at $-20^{\circ} \mathrm{C}$ overnight. The bilayer mixture was freezedried (CHRIST, Alpha 1-2 LDplus, UK) at $-50^{\circ} \mathrm{C}$ for $48 \mathrm{~h}$.

\section{Physical and chemical characterization of membranes}

The physical and chemical properties of the bilayer membrane were investigated by using different analytical techniques.

Scanning electron microscopy. The surface morphology and porous structure were examined using scanning electron microscopy (SEM), TESCAN Vega3 LMU. Before analysis, the membranes were gold coated for $90 \mathrm{~s}$ through a sputter coater (QUORUM). The pore 
size was later quantified by using a line-drawing feature of ImageJ software. The diameter of both small and large pores on the images was measured manually and then the mean pore size was determined as a mean area (measured by software) of six straight lines for each image.

Fourier transform infrared spectroscopy. The structural changes due to bonding after lyophilization were investigated using Fourier transform infrared spectroscopy (FTIR) (Thermo Nicolet 6700, USA) in conjunction with attenuated total reflectance sampling accessory equipped with germanium crystal. Spectra were obtained at $4 \mathrm{~cm}^{-1}$ resolution, accumulating 256 number of scans within mid-infrared (IR) range (4000-550 $\mathrm{cm}^{-1}$ ).

$X$-ray diffraction. The phases of BG in bilayer composite membranes were studied by X-ray diffractometer (XPERT-PRO, PANalytical), operated at $40 \mathrm{kV}$ and $40 \mathrm{~mA}$ using $\mathrm{Cu} \mathrm{K} \alpha$ radiation. Wide-angle X-ray diffraction (XRD) patterns were made over an angle range of $2 \theta=10^{\circ}-60^{\circ}$ at $0.02^{\circ}$ step size.

\section{Swelling studies}

The swelling ability of the membranes $(1 \mathrm{~cm}$ length $\times 1$ $\mathrm{cm}$ width) was measured in phosphate-buffered saline (PBS). Pre-weighed samples were dipped in PBS $(5 \mathrm{~mL})$ at $37^{\circ} \mathrm{C}$ temperature for periodic time intervals, i.e. 0 , $15,30,60,120,140$, and $160 \mathrm{~min}$. After each time interval, samples were taken out from PBS, and excess water was removed by squeezing out between two sheets of filter paper prior to weighing the samples. The swelling percentage was measured using the formula:

$$
\text { Swelling } \%(\mathrm{Q})=\left(\mathrm{w}_{\mathrm{w}}-\mathrm{w}_{\mathrm{d}}\right) / \mathrm{w}_{\mathrm{d}} \times 100
$$

where $\mathrm{w}_{\mathrm{d}}$ and $\mathrm{w}_{\mathrm{w}}$ represent the weight of dry and wet sample, respectively. The swelling ratio was obtained in triplicate and expressed as mean \pm standard deviation (SD).

\section{Cell culture}

Human carcinoma cell line (MG63, ECACC general cell collection) was maintained in Dulbecco's modified Eagle's medium (Biosera, UK) added with fetal calf serum (10\%, Sigma-Aldrich, UK), 1-glutamine ( $2 \mathrm{mM}$, Sigma-Aldrich, UK), penicillin $(100 \mathrm{U} / \mathrm{mL}$, SigmaAldrich, UK), streptomycin $(1.2 \mu \mathrm{g} / \mathrm{mL}$, SigmaAldrich, UK), and amphotericin B $(0.0625 \mu \mathrm{g} / \mathrm{mL})$. Cells were cultured in T75 tissue culture flask to $90 \%$ confluence in an incubator (at $37^{\circ} \mathrm{C}$ temperature under
$5 \% \mathrm{CO}_{2}$ atmosphere, where media was changed after every two to three days) and passaged using trypsinEDTA (Sigma-Aldrich, UK). The passage number was kept between 60 and 65. Membranes were sterilized using 70\% ethanol (Sigma-Aldrich, UK) for overnight incubation $(\leq 14 \mathrm{~h})$ and washed with PBS (three times, $15 \mathrm{~min}$ each) to eliminate any residual ethanol, followed by dipping in cell culture medium for $1 \mathrm{~h}$ before plating them on 96-well plate for cell seeding

Alamar blue assay. The cytotoxic response of membrane was determined using Alamar blue assay on human osteosarcoma cell line (MG63). Briefly, the sterilized membranes were put in 96-well plate, and then MG63 cells were seeded at an initial cell density of 20,000 cells/ well. The cell viability was quantified after 24,48 , and $72 \mathrm{~h}$. At each end-point, medium for cell culture was removed and replaced with Alamar blue solution (Sigma-Aldrich, UK working solution at the $100 \mu \mathrm{M}$ solution in serum-free media) for $4 \mathrm{~h}$ at $37^{\circ} \mathrm{C}$ temperature. Fluorescence plate reader (Bio-TEK, NorthStar Scientific Ltd. UK) was used to measure the fluorescence at $570 \mathrm{~nm}$. Following the measurements, the samples were washed with PBS, and fresh serumcontaining cell culture medium was added to incubate until next end-point. The above method was repeated at every end-point. The experiment was done in triplicates to obtain a mean value $\pm \mathrm{SD}$. The mean values of absorbance from each scaffold were plotted over a graph against various end-points.

Immuno-staining. The membranes were sterilized as mentioned above and plated in 96-well plate. Human osteosarcoma cell line MG63 was grown on either surface of the membrane separately with an initial seeding density of 20,000 cells/well. Then, these membranes were incubated for three end-points $(2,6$, and 10 days) at $37^{\circ} \mathrm{C}$ temperature under $5 \% \mathrm{CO}_{2}$ atmosphere. Cell culture medium was replenished every two to three days with fresh medium. After each endpoint, washing of scaffolds was done using PBS, and then $3.7 \%$ formaldehyde (30 min) was used for cells fixation and $0.1 \%$ Triton X-100 for permeabilization (for $30 \mathrm{~min}$ ). Phalloidin-tetramethyl rhodamine B isothiocyanate (TRITC) was used for immunofluorescent staining of F-actin filaments $(10 \mu \mathrm{g} / \mathrm{mL}, 2 \mathrm{~h})$ and $4^{\prime}, 6-$ diamidino-2-phenylindole (DAPI) (300 $\mathrm{nM}, 15 \mathrm{~min})$ for nuclear staining. Membranes were washed with PBS after each fluorescent probe incubation. Finally, the membranes were left in PBS for imaging. The images were taken by Zeiss confocal microscopy, phalloidin TRITC was excited at $\lambda \mathrm{ex}=543 \mathrm{~nm}$; and emission at $\lambda \mathrm{em}=565-615 \mathrm{~nm}$, DAPI was excited using coherent Chameleon pulsed IR multiphoton laser at $800 \mathrm{~nm}$ and emission detected at 435 to $485 \mathrm{~nm}$. 
Images were taken using Achroplan water dipping objective $(10 \times$, NA 0.3 , WD 3.1$)$ from each seeding surface of the membrane, and the data were processed using Zeiss LSM image browser.

\section{Animal studies}

Animal experiments were conducted according to the instructions and permission from the animal welfare and ethical committee of University of Health Sciences, Lahore, Pakistan. The institutional guidelines for the care and use of animals were followed in this study. Animal studies were performed on wild-type male adult rats (two months old) weighing 140 to $180 \mathrm{~g}$. Rats were bred in the animal facility of University of Health Sciences, Lahore, Pakistan. Animals were kept in specific pathogen-free, clean, and well-aerated individual cages with an optimum supply of light, food, and water. Experiments were performed independently three times on each animal of the experimental groups and two times on each animal of the control groups. In order to reduce the overall sufferings and number of animals, four membranes were implanted subcutaneously in a single animal. Experimental as well as control animal groups were further divided into two subgroups: (i) experimental and control groups observed at $48 \mathrm{~h}$ after implantations and (ii) experimental and control groups observed at six weeks after implantation. For implantations in experimental groups, animals were first anesthetized with intraperitoneal injections of xylazine hydrochloride $(20 \mu \mathrm{L})$ and ketamine $(300 \mu \mathrm{L})$. Anesthetized animals were then shifted to a clean bench, and fur from the dorsal side was removed using a hair trimmer. Eye ointment polyfex (polymyxin B and bacitracin; GlaxoSmithKline, UK) was then applied on eyes to avoid dryness and infection. Four longitudinal surgical incisions $(1 \mathrm{~cm}$ each) were made at different locations on the dorsal side of the animal, and subcutaneous pouches were generated through these incisions. Four sterilized membranes with the dimensions of $1 \times 1 \mathrm{~cm}^{2}$ were then inserted into these subcutaneously generated pouches (supporting information, Figure S3). After implantation, surgical wounds were closed with silk suture material (black braided silk suture 3/0,1/2 circle curved cutting $30 \mathrm{~mm}$ (care)), and Pyodine-Iodine solution (an antiseptic used for skin disinfection) was then applied on wound openings. While, in the control group, four surgical pouches were generated in each animal according to the abovementioned protocol; however, they were closed with suture material without any implantations. Health status and weight of all animals before and after the start of experiments were regularly monitored. After the completion of selected time intervals, animals were peacefully euthanized by $\mathrm{CO}_{2}$ asphyxiation followed by cervical dislocation. Implanted membranes together with tissue were then removed. The obtained membranes were fixed in $4 \%$ paraformaldehyde and processed for further histological examination.

Histology of tissue-implant interface. The tissues were fixed in $4 \%$ paraformaldehyde and dehydrated with an increasing concentration of ethanol: $70 \%, 85 \%, 95 \%$, and $100 \%$ for $1 \mathrm{~h}$ in order to avoid tissue distortion. After dehydration, tissues were dipped in a pre-melted wax $\left(70^{\circ} \mathrm{C}\right)$ and fixed in the form of the block for $1 \mathrm{~h}$. The blocks were placed in a refrigerator at $-20^{\circ} \mathrm{C}$ for $30 \mathrm{~min}$ for adequate solidification and clamped in the carrier of the microtome and trimmed to acquire tissue sections of $5 \mu \mathrm{m}$. These tissue sections were transferred to slides subjected to hematoxylin and eosin staining, whereby tissue samples were dipped in xylene I and xylene II for $5 \mathrm{~min}$ and rehydrated by dipping in $100 \%$ (absolute ethanol), $80 \%, 70 \%$, and $50 \%$ ethanol (for $1 \mathrm{~min}$ each). Then, samples were dipped in deionized water for $30 \mathrm{~min}$ and soaked in hematoxylin for $3 \mathrm{~min}$. Excess stain was washed with tap water for $30 \mathrm{~min}$ followed by a fast dip in $0.5 \%$ acidic alcohol. The samples were placed under running water for $1 \mathrm{~min}$ and dipped in $1 \%$ ammonia solution for $1 \mathrm{~min}$ followed by immersion in $50 \%$ alcohol for $1 \mathrm{~min}$ and eosin for half an hour. Then, samples were soaked again in $50 \%, 70 \%$ and $80 \%$ ethanol for $10 \mathrm{~s}$, in $100 \%$ ethanol for $2 \mathrm{~min}$ and in xylene for $1 \mathrm{~min}$. The samples were dried and mounted with mixture of Distyrene, Plasticizer (tricresyl phosphate), and Xylene (DPX) mounting media, and images were taken using a fluorescent microscope $\left(\mathrm{VWR}^{\circledR}\right.$ Inverted Fluorescence Microscope 89404-464, US) at varying resolutions.

Statistical analysis. For swelling and in vitro cell studies, statistical analysis was performed with one-way analysis of variance, post hoc Tukey's test. Furthermore, repeated measure analysis was also performed for swelling study only. A p-value of $<0.05$ was considered significant, and tests were performed by using SPSS version 22 (IBM, USA).

\section{Results}

\section{Physical and chemical properties}

Macro and microstructure (SEM) analysis. The objective of this research was to develop a bilayer membrane with the non-porous upper layer to prevent intrusion of epithelial cells and porous lower layer to maintain blood vessels and nutrient mass transfer. The pictorial images of single PU film, single PCL-BG layer, and bilayer 
(PU/PCL-BG) membrane, respectively, are shown in supporting information (Figure S1(a) to (c)). The thickness of the bilayer membrane was about $1.85 \mathrm{~mm}$.

The topography and microstructure of single and bilayer membranes were observed by SEM (Figure 1). Figure 1(a) to (c) shows the topography of pure PCL layer and the PCL-BG composite layers prepared by the freeze-drying method. A clear hierarchically arranged pattern was observed with two different sized pores, with the large $(26-38 \mu \mathrm{m}$ in diameter, mean diameter $33 \pm 5$ ) and small interconnected pores (2-3 $\mu \mathrm{m}$ diameter), in the pure PCL (supporting information, Figure S4 (c) and (d)). The porous structure was symmetrical throughout the membrane. Addition of $B G$ in the PCL layer changed the pattern and shape of pores. Figure 1(e) shows the asymmetrical pores with wide openings $(22-65 \mu \mathrm{m}$ pore diameter, mean diameter $37 \pm 22$ ) in the PCL-BG layer (supporting information S4(e)). The change in the pattern could be related to the existence of a biphasic system (PCL and $\mathrm{BG}$ ) and density difference. Figure $1(\mathrm{f})$ shows that the BG nanoparticles are embedded in the PCL polymer. The distribution of these nanoparticles was uniform and homogeneous.

Microscopic image of the pure PU membrane is presented in Figure 1(g) to (i), which showed the nonporous structure of the PU in the bilayer membrane. Micrographs of the intersection of the two layers (Figure 1(k) and (1)) show a good interphase or attachment between the two layers, obtained by using a small amount of the same solvent used for processing in both the polymeric systems. No detachment was observed throughout the surface. However, at certain points, the lacy/fibrous structure was observed which was continuous and penetrating and hence contributes to intact structure formation. No delamination was observed during 28 days of soaking in PBS (not shown in results) which also showed that bilayer structure was stable. The membrane was flexible but not fragile, and the interface shows that two layers intermingled with each other. The results confirmed that a good interface between two layers was achieved by manipulating the number of solvents between the different polymeric layers.

Fourier transform infrared spectroscopy. Chemical structural properties of the PCL, the PU, and the PCL-BG composite membranes were studied by FTIR spectroscopy to identify the chemical structure of the backbone of polymeric molecules and functional groups that orientated themselves to the surface of the molecule. In FTIR spectrum (Figure 2(a)) of the PCL-BG composite layer, characteristic vibrational spectral peaks of PCL and BG were observed. Asymmetric and symmetric $-\mathrm{CH}_{2}$ stretching vibration peaks were observed at
$2944 \mathrm{~cm}^{-1}$ and $2861 \mathrm{~cm}^{-1}$, and the peaks at $1190 \mathrm{~cm}^{-1}$, $1152 \mathrm{~cm}^{-1}$, and, $1170 \mathrm{~cm}^{-1}$ were attributed to symmetric stretching of $\mathrm{C}-\mathrm{O}-\mathrm{C}, \mathrm{C}-\mathrm{O}$, and $\mathrm{C}-\mathrm{C}$, respectively. The spectral peaks at $1240 \mathrm{~cm}^{-1}, 1288 \mathrm{~cm}^{-1}$, and 1714 $\mathrm{cm}^{-1}$ were attributed to $\mathrm{C}-\mathrm{O}-\mathrm{C}$ stretching vibrations and $\mathrm{C}-\mathrm{O}$ bonds. ${ }^{17}$ The characteristic vibrational spectral peaks of the BG appeared in FTIR spectrum of the PCL-BG composite membrane. A broad band with a shoulder at $1290-890 \mathrm{~cm}^{-1}$ was specific to symmetric and asymmetric stretching of $\mathrm{P}-\mathrm{O}$ and $\mathrm{Si}-\mathrm{O}-\mathrm{Si}$ of the BG. ${ }^{18,19}$ A small band (appeared as shoulder) of $\mathrm{Si}-\mathrm{O}-$ NBO (non-bridging oxygen) for $\mathrm{Si}-\mathrm{O}-\mathrm{M}$ (where $\mathrm{M}=\mathrm{Ca}^{+2}$ ) bonds was observed around $960 \mathrm{~cm}^{-1} \cdot{ }^{20} \mathrm{~A}$ broad band between 840 and $760 \mathrm{~cm}^{-1}$ was assigned to bending $\mathrm{Si}-\mathrm{O}-\mathrm{Si}$ mode. The $\mathrm{O}-\mathrm{P}-\mathrm{Ca}$ bond appeared at $660 \mathrm{~cm}^{-1} \cdot{ }^{21}$ There was a slight shift in peaks due to the interaction between the $\mathrm{BG}$ and the PCL in the composite.

In the FTIR spectrum of the PU layer, the characteristic band of free carbonyl $(\mathrm{C}=\mathrm{O})$ group appeared as a shoulder at $1726 \mathrm{~cm}^{-1}$ and bonded $\mathrm{C}=\mathrm{O}$ at $1696 \mathrm{~cm}^{-1}$. The symmetric and asymmetric stretching vibrations of $\mathrm{C}-\mathrm{H}$ with carbonyl appeared at $2845 \mathrm{~cm}^{-1}$ and $2938 \mathrm{~cm}^{-1}$, respectively, and bending peaks of $\mathrm{C}-\mathrm{H}$ were observed at $1348 \mathrm{~cm}^{-1}$. The stretching band of $\mathrm{N}-\mathrm{H}$ was observed at $3331 \mathrm{~cm}^{-1}$, whereas the $1598 \mathrm{~cm}^{-1}$ peak attributed to the $\mathrm{C}=\mathrm{C}$ vibrational benzene ring, and $1539 \mathrm{~cm}^{-1}$ and $1224 \mathrm{~cm}^{-1}$ were the $\delta(\mathrm{N}-\mathrm{H})+$ vibrational $(\mathrm{C}-\mathrm{N})$ peaks. The strong $\delta(\mathrm{N}-\mathrm{H})+v(\mathrm{C}-\mathrm{N}), \quad \beta(\mathrm{C}-\mathrm{H})$ peak appeared at $1311 \mathrm{~cm}^{-1}$. $^{22}$

$X$-ray diffraction. The phase development of the BG in bilayer membrane was investigated by XRD studies. Figure 2(b) shows the XRD pattern of the upper and lower surface of the bilayer composite membrane. The preparation process did not change the phases of the BG, but there was suppression of few peaks due to the amorphous nature of polymeric phases. ${ }^{16}$

Swelling properties. The swelling ratio of the GTR/GBR membrane was studied up to a period of $160 \mathrm{~min}$ (Figure 3(a)). PCL-BG membrane showed maximum swelling of $140 \%$ compared to the PU and bilayer PU and PCL-BG membrane after $160 \mathrm{~min}$. The bilayer membrane absorbed the least amount of water and showed a swelling percentage of only $77 \%$. A variable swelling behavior was observed for the PU membrane, which might be due to its hydrophobic nature. The post hoc Turkey's test showed that there was the insignificant difference $(p>0.05)$ among the groups except for PU-PCL at 160 min which showed a significant difference $(p<0.05)$ with PCL/BG and PU. Whereas repeated measure analysis showed the non-significant difference $(p>0.05)$ with respect to time intervals. 

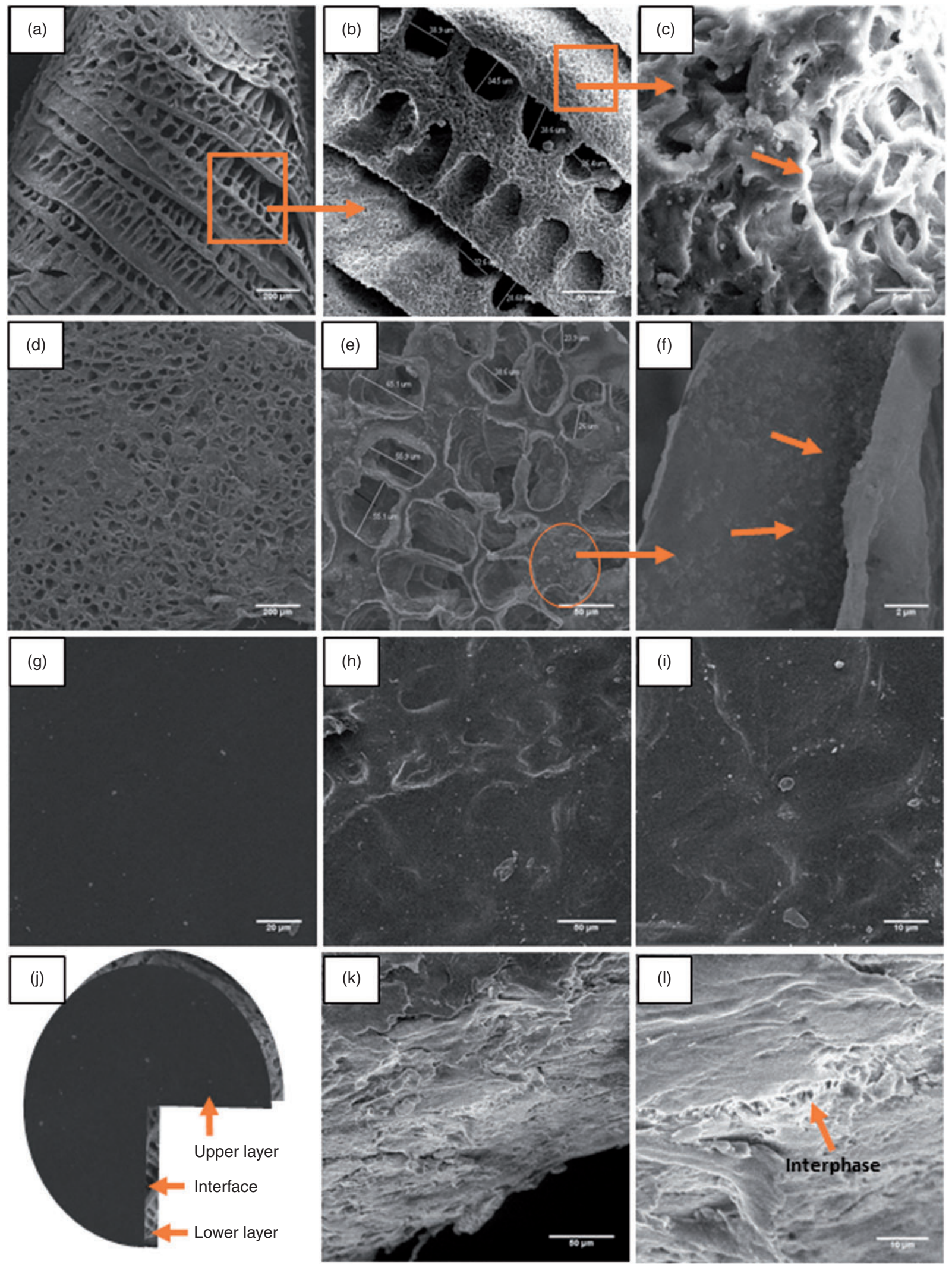

Figure I. (a-c) SEM images of pure PCL, $(d-f)$ PCL-BG layer in bilayer membrane, $(g)$ pure PU, (h and i) PU layer in bilayer membrane, (j) bilayer membrane, and $(\mathrm{k}$ and $\mathrm{l})$ interface of composite membranes. 
(a)

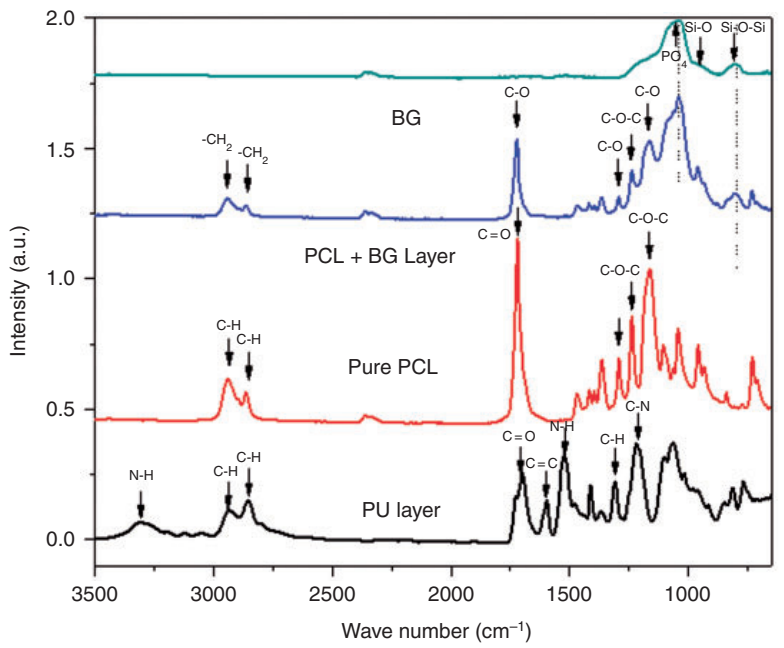

(b)

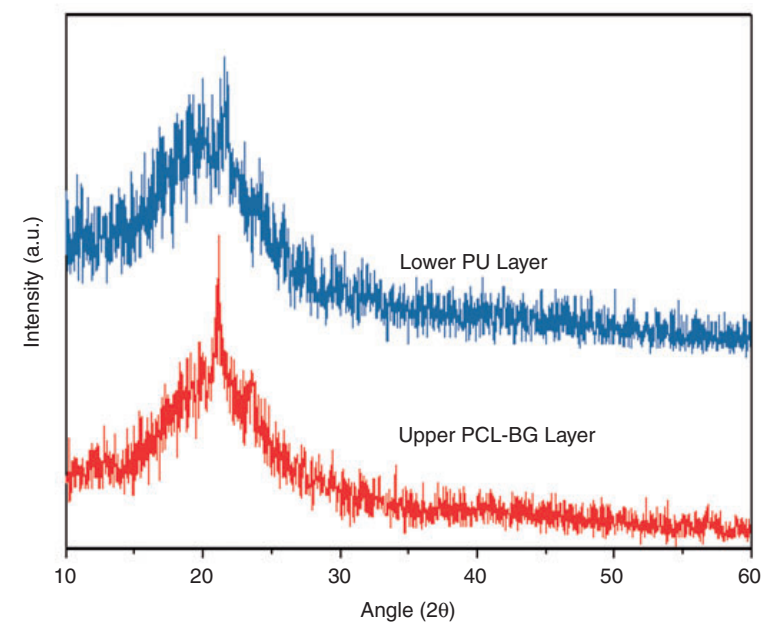

Figure 2. FTIR spectrum of PU, BG, PCL, and PCL-BG (a) and XRD pattern of lower and upper surface of bilayer membrane (b). PCL: polycaprolactone; BG: bioactive glass; PU: polyurethane.

(a)

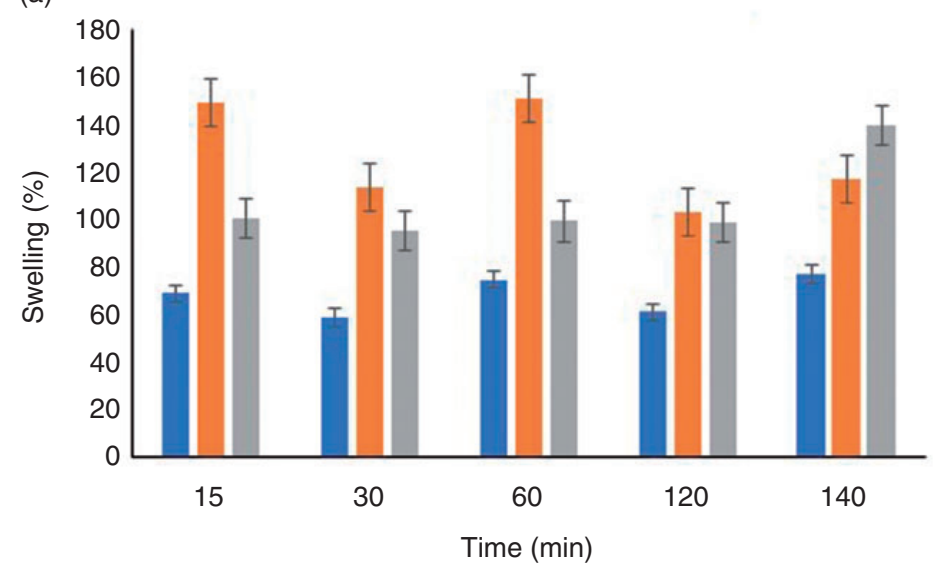

Bilayered membrane

a $\mathrm{PU}$

PCL-BG

(b)

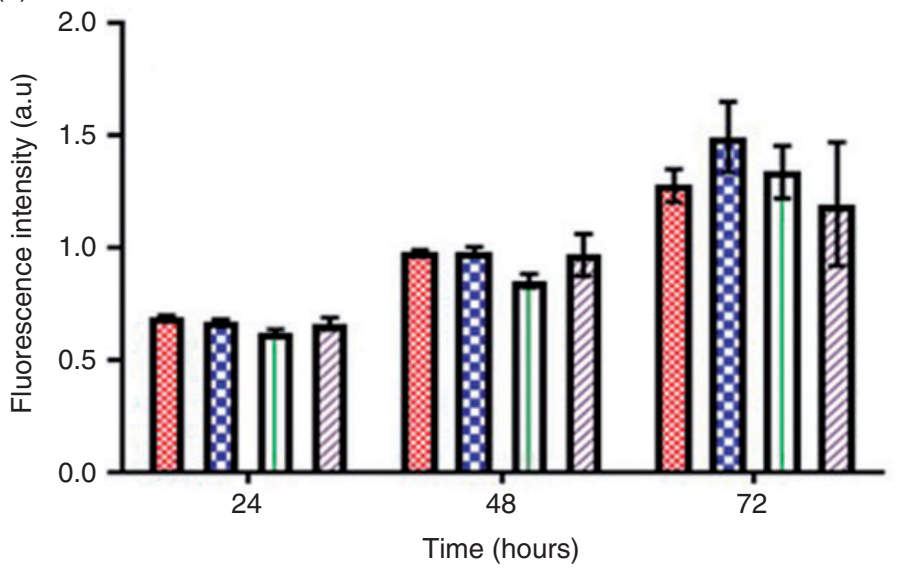

ख PU membrane

PCL-BG membrane

III Bilayered membrane

TCP

Figure 3. Swelling studies of single layer (PU, PCL-BG) and bilayer GTR/GBR membranes (a) and metabolic activity of MG63 osteosarcoma cells (cultured for 24, 48 and $72 \mathrm{~h}$ ) in the membranes measured using Alamar blue (b). The value represents the mean absorbance \pm standard deviation. PCL: polycaprolactone; BG: bioactive glass; PU: polyurethane; TCP: tissue culture plate. 


\section{Cell studies}

Alamar blue. The cell viability was evaluated by direct cell Alamar blue assay, which also showed the cell's proliferation and metabolic activity as shown in Figure 3(b). Metabolic activity of osteosarcoma cells MG63 increased with time in all membranes, suggesting an increase in cellular viability without any cytotoxic interference of membranes over $72 \mathrm{~h}$ in culture. No statistically significant cytotoxicity was noticed in any of the membrane (in all three end-points) when compared with the cell in TCP (tissue culture plate, control). The PCL-BG membrane showed significance cell proliferation over TCP (at $72 \mathrm{~h}$, p-value $=0.0027$ ).

Immunostaining. Osteosarcoma cell line was seeded on either surface of the pure PU, the PCL-BG, and the bilayer membranes. Phalloidin TRITC and DAPI staining were used to observe cell adherence to membranes. The immunostaining of cells seeded on either surface of the PU membrane after $48 \mathrm{~h}$ showed consistent growth of MG63 over the whole surface of the membrane (Figure S2 supporting information). Almost equal cell attachment was observed on either surface of the PU membrane after initial seeding. Figure 4 shows cell growth and attachment on the surface of the PCL-BG membrane at 2, 6, and 10 days. It was evident that the number of cells increased with the passage of time in the PCL-BG layer. Cells attachment and proliferation were also studied on either surface of the bilayer membrane (Figure 5) at different end-point
(2, 6, and 10 days). The PCL-BG surface cell seeding showed more cell attachment than the PU surface. The number of cells on the PCL-BG was increased rapidly at days 6 and 10, while this number almost remained unchanged on the PU side. Figure 5 shows that the cells were also attached inside the pores of the PCL-BG. The more red color seen on day 2 (Figure 5) was due to background staining (biomaterial staining), as the material had an increased auto-fluorescence from TRITC emission. On contrary, the bottom side (the PU layer) was non-porous, and most of the cells died on its surface. The PU membrane is a flat twodimensional surface, while the PCL-BG membrane has pores and therefore provided more surface area for cells to proliferate than the PU membrane. This was evident from Figures 4 and 5 which indicate an increased progressive growth of cells in the PCL-BG membrane as compared to the PU membrane.

\section{In vivo studies}

In vivo studies by using animals are required prior to the exploitation of any developed materials for clinical application. Therefore, bilayer membranes were subcutaneously inserted into rats to investigate host tissue responses. The subcutaneous tissue was selected for implantation since it avoids complex surgical procedures and represents real-time host-material interactions. It allows rapid and reliable observation of gross inflammatory host responses, such as swelling and redness (Figure S3 supporting information).

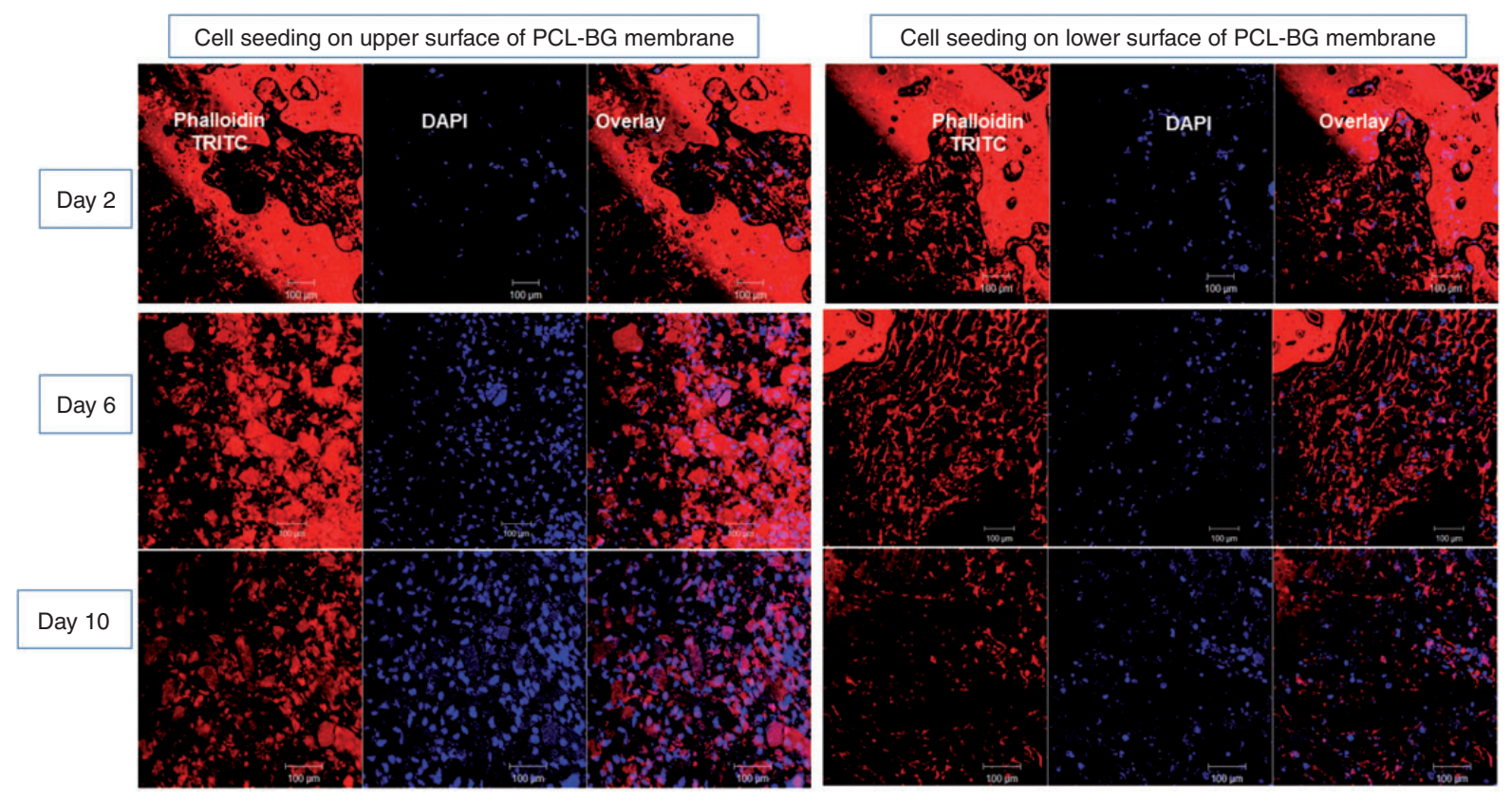

Figure 4. Cell attachment and proliferation on PCL-BG membrane observed over 10 days in culture. DAPI: 4',6-diamidino-2phenylindole; TRITC: tetramethyl rhodamine B isothiocyanate; PCL: polycaprolactone; BG: bioactive glass. 


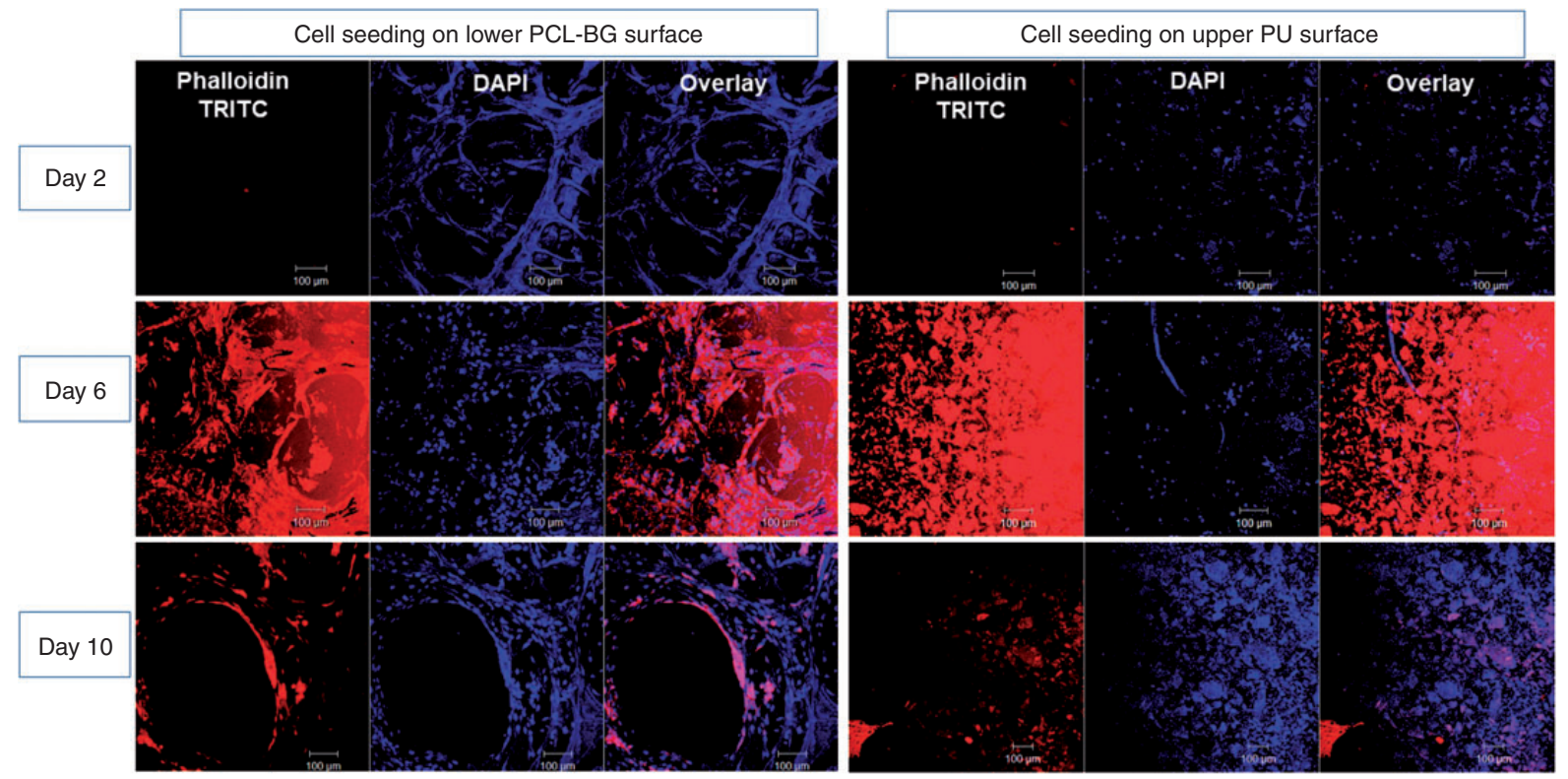

Figure 5. Cell attachment and proliferation on bilayer membranes observed over 10 days in culture imaged. DAPI: 4',6-diamidino-2phenylindole; TRITC: tetramethyl rhodamine B isothiocyanate; PCL: polycaprolactone; BG: bioactive glass; PU: polyurethane.
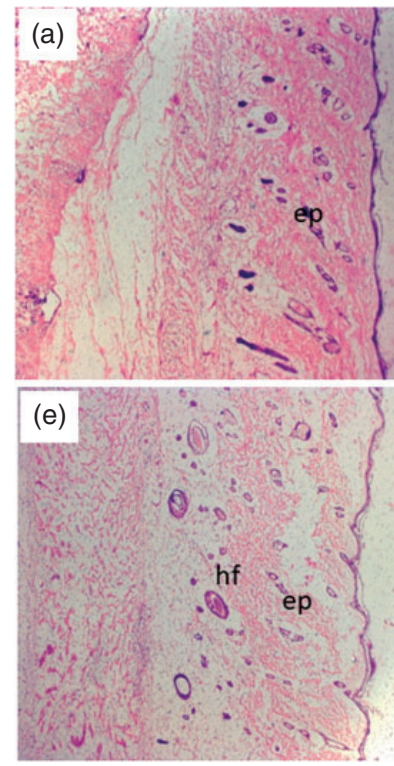
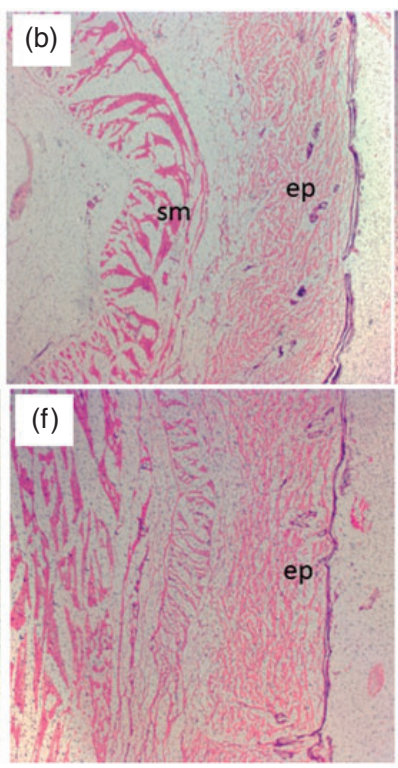

(c)
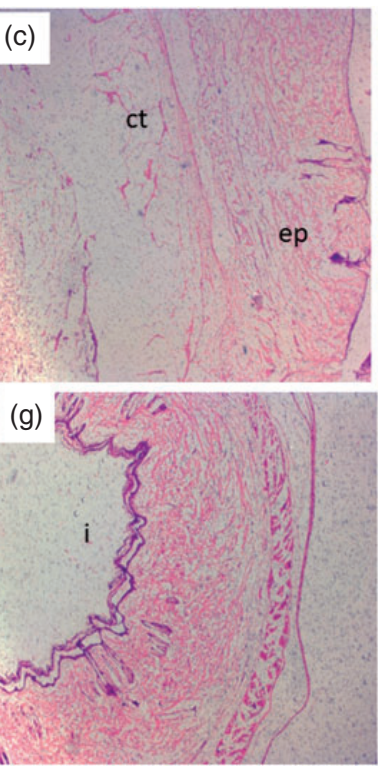
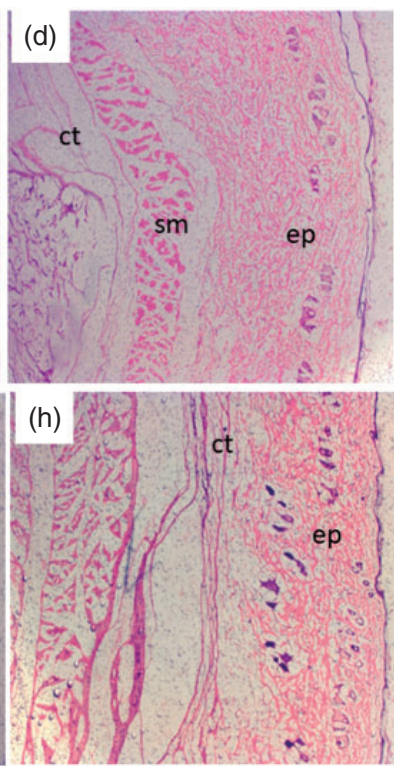

Figure 6. Histological evaluation of implant-tissue interface ( $a$ and $b=$ control group, $c$ and $d=$ bilayer membrane, e and $f=P U$, and $\mathrm{g}$ and $\mathrm{h}=\mathrm{PCL}-\mathrm{BG}$ membranes). Membranes were subcutaneously implanted into rats and followed by $48 \mathrm{~h}$ and six weeks of implantation, membranes together with adjacent tissue were fixed and subjected to hematoxylin and eosin stain. Tissue responses towards bilayer membranes after $48 \mathrm{~h}$ of implantation (left side) and after six weeks of implantation (right side). i: site of implantation; ep: epidermis; ct: connective tissue; sm: skeletal muscles; hf: hair follicle.

Results from visual inspection did not reveal any signs of inflammation, as no swelling or redness at the implantation site was observed (Figure S3, supporting information). Moreover, there were no indications of bacterial contamination or infections, such as pus accumulation at the implantation site. During the entire study period, animals remained healthy exhibiting normal walking, feeding, and drinking behaviors, thereby indicating biocompatible properties of bilayer membranes. Detailed implant-tissue interactions were further evaluated by applying histological analysis. After six weeks of implantation, a connective tissue 
layer indicative for good material-tissue interaction was visible around all membranes (Figure S3(d), supporting information). Histological analysis of tissue adjacent to bilayer membranes indicated no signs of inflammation or accumulation of host immune cells except normal foreign body responses, which was similar to controls (Figure S2, supporting information) (Figure 6). Overall, the absence of host immune cells, as well as consistency in tissue adjacent to implanted membranes, was indicative of good compatibility of membranes for prospective clinical applications.

\section{Discussion}

The cell occlusivity is a major prerequisite for the GTR/GBR membranes. It requires appropriate membrane surface structure to prevent infiltration of epithelial cells from surroundings while maintaining the essential mass transference through the membrane. In the present study, the morphology, adhesion, and pore structure of the GTR/GBR membrane were controlled by using the multi-solvent system. It has been reported that in freeze-drying, liquid-liquid miscibility plays an important role in porosity, densification, and morphology of materials. ${ }^{23-25}$ Therefore, a mixture of solvents with good miscibility and delayed liquid-liquid phase separation was used to fabricate a membrane with dense non-porous structure. Binary solvent system, comprised of DMF and dioxane, was selected for dissolution and fabrication of non-porous PU layer. DMF and dioxane have relatively better miscibility and separate very slowly (liquid-liquid phase separation) during cooling and lyophilization, subsequently avoiding the formation of pores or cracks. Therefore, a dense non-porous PU structure was formed after lyophilization. Few macro-pores observed on the surface of the PU layer (at low magnification) were due to percolation of the solvent during the pouring process; however, at high magnification, the structure was found to be non-porous. In the lower layer (PCL-BG), porous structure with a different pattern (Figure 1(e)) was observed as compared to the pure PCL layer (Figure 1(b)). This change in morphology was due to the presence of BG nanoparticles, which acted as filler and changed the freezing pattern of the PCL. These pores had a diameter of 22 to $65 \mu \mathrm{m}$ and helped in maintaining essential mass transference through the membrane during regeneration of bone and tissues. The use of the same solvent (dioxane) in both layers resulted in the formation of firm adhesion between the two layers. The two membranes were not detached during different physical and biological investigations.

It has been reported that the structural morphology, such as porosity and surface area of membranes, affects the physical and biological properties. ${ }^{26}$ In this study, the PCL-BG layer possessed porous structure and therefore exhibited enhanced swelling kinetics. Swelling behavior is also a function of surface area and therefore increased surface area due to the presence of the $\mathrm{BG}$ in the PCL membrane might be the second factor for increased water absorption for the PCL-BG membranes. The mono-layered PU membrane showed poor swelling due to non-porous structure and hydrophobic nature. The bilayer membrane showed less swelling than the single PCL-BG membrane. Interconnectivity of porous structures plays an important role in water absorption of the membranes ultimately affecting the physical properties of the designed materials, as the bilayer membrane showed less swelling than the single PCL-BG membranes.

The most important criterion for new biomaterials before their prospective clinical application is their biocompatibility. Therefore, synthesized membranes were investigated for their biocompatibility and immune responses both in vitro and in vivo. In bilayer membranes, the cells were evenly spread over the surface of membranes. The enhanced cell attachment and proliferation on the lower layer (PCL-BG surface) was due to enhanced surface area and roughness because of the presence of the BG nanoparticles and porous structure. The bioactive materials, specifically the $\mathrm{BG}$, promote the cellular processes and improve cell adhesion and proliferation. ${ }^{27}$ An increased cell metabolic activity over time with a progressive increase of cell growth and attachment through the porous structure of the PU-BG surface of the membrane was observed. More cell attachment was observed inside the porous structure of the PCL-BG as compared to the surface, which is necessary for the GTR/GBR membrane. ${ }^{28}$ The number of cells on upper side (the PU surface) of the membrane was far less than the PCL-BG surface due to hydrophobic properties of $\mathrm{PU}$, which is in accordance to the requirement of the GTR/GBR membranes. In the GTR/GBR membranes, the upper surface is non-porous and hydrophobic to avoid the attachment and down-growth of epithelial and connective tissues into the defect, while lower surface should promote the cells attachment and bone regeneration. In vitro cell compatible properties of bilayer membranes were further validated in the small animal model. All membranes were implanted subcutaneously in soft tissue to allow maximum contact between host immune systems and implanted material. ${ }^{29}$ The rats were healthy and grew normally after implantation. No inflammatory responses were observed during implantation and surgical procedures which were observed for a period of six weeks. The population of immune cells in tissue samples adjacent to membranes was similar to controls after a short or long period of implantation suggesting 
good biocompatibility which was evident from favorable tissue response of membranes. Bacterial colonization and subsequent biofilm formation remain a major challenge for the in vivo performance of novel materials. Interestingly, no signs of bacterial contamination, such as accumulation of pus, could be observed near implanted membranes. The previously reported studies showed the optimal persistence of the GTR membranes in vivo vary from four to several weeks. ${ }^{30}$ Thus, the controlled morphology and biocompatibility both in in vitro and in vivo studies of the bilayer membranes confirmed that the bioactive membranes developed in this study have excellent potential to be used for wound healing and tissue regeneration applications.

\section{Conclusion}

This study has established a concept design of the bilayer GTR/GBR membrane with the upper nonporous hydrophobic PU layer and the lower porous hydrophobic PCL-BG layer by modified lyophilization method. The pores or crack formation in the upper layer was avoided using DMF and dioxane solvents with high miscibility and low liquid-liquid phase separation. This design prevents the penetration and growth of epithelial cells and connective tissues into the wound and facilitates the growth and attachment of cells and transport of nutrition (lower surface) at the wound site. The bilayer structure developed in this study demonstrated excellent biocompatibility and supported the growth of cells towards lower surface. The introduction of the $\mathrm{BG}$ nanoparticles into the PCL increased the stiffness of the membrane and the increased cell attachment and proliferation. In vivo studies revealed that the bioactive membranes did not cause any inflammatory response, and fast healing was observed as compared to control, which can be contributed to the bioactivity of the glass nanoparticles impregnated within the polymeric network structure.

\section{Authors' Note}

Asma Tufail is also affiliated with Department of Material Science and Engineering, Kroto Research Institute, The University of Sheffield, Sheffield, United Kingdom and Fachgebiet Keramische Werkstoffe / Chair of Advanced Ceramic Materials, Institut für Werkstoffwissenschaften und -technologien, Technische Universität Berlin, Hardenbergstr, Berlin, Germany.

\section{Acknowledgements}

We acknowledge Higher Education of Pakistan and Ministry of Science and Technology Pakistan for providing analytical facilities to Interdisciplinary Research Centre in Biomedical Materials, COMSATS University Islamabad, Lahore
Campus, Pakistan. We are also grateful to Alexander von Humboldt for facilitating us in this project.

\section{Declaration of Conflicting Interests}

The author(s) declared no potential conflicts of interest with respect to the research, authorship, and/or publication of this article.

\section{Funding}

The author(s) received no financial support for the research, authorship, and/or publication of this article.

\section{ORCID iD}

Abdul Samad Khan (D) http://orcid.org/0000-0002-2165$800 \mathrm{X}$

Asma Tufail (D) http://orcid.org/0000-0001-9624-1842

\section{Supplemental Material}

Supplemental material for this article is available online.

\section{References}

1. Jamuna-Thevi K, Saarani NN, Abdul Kadir MR, et al. Triple-layered PLGA/nanoapatite/lauric acid graded composite membrane for periodontal guided bone regeneration. Mater Sci Eng C 2014; 43: 253-263.

2. Qasim SB, Delaine-Smith RM, Fey T, et al. Freeze gelated porous membranes for periodontal tissue regeneration. Acta Biomaterialia 2015; 23: 317-328.

3. Qasim SB, Husain S, Huang Y, et al. In-vitro and in-vivo degradation studies of freeze gelated porous chitosan composite scaffolds for tissue engineering applications. Polym Degrad Stab 2017; 136: 31-38.

4. Sun X, Xu C, Wu G, et al. Poly (lactic-co-glycolic acid): applications and future prospects for periodontal tissue regeneration. Polymers 2017; 9: 189.

5. Sheikh Z, Khan AS, Roohpour N, et al. Protein adsorption capability on polyurethane and modifiedpolyurethane membrane for periodontal guided tissue regeneration applications. Mater Sci Eng C Mater Biol Appl 2016; 68: 267-275.

6. Wissing TB, Bonito V, Bouten CVC, et al. Biomaterialdriven in situ cardiovascular tissue engineering - a multidisciplinary perspective. NPJ Regen Med 2017; 2: 18.

7. Gürbüz S, Demirtaş TT, Yüksel E, et al. Multi-layered functional membranes for periodontal regeneration: Preparation and characterization. Mater Lett 2016; 178: 256-259.

8. Tsui YK and Gogolewski S. Microporous biodegradable polyurethane membranes for tissue engineering. J Mater Sci Mater Med 2009; 20: 1729-1741. Journal Article. DOI: $10.1007 / \mathrm{s} 10856-009-3722-4$.

9. Khan AS, Aziz MS, Paul D, et al. Synthesis and in-vitro analysis of degradative resistance of a novel bioactive composite. J Bionanosci 2008; 2: 75-88. 
10. Zahid S, Shah AT, Jamal A, et al. Biological behavior of bioactive glasses and their composites. RSC Adv 2016; 6: 70197-70214.

11. Hench LL. The story of Bioglass ${ }^{\circledR}$. J Mater Sci Mater Med 2006; 17: 967-978.

12. Srinivasan S, Jayasree R, Chennazhi KP, et al. Biocompatible alginate/nano bioactive glass ceramic composite scaffolds for periodontal tissue regeneration. Carbohydr Polym 2012; 87: 274-283.

13. Sheikh Z, Qureshi J, Alshahrani AM, et al. Collagen based barrier membranes for periodontal guided bone regeneration applications. Odontology 2017; 105: 1-12.

14. Zhang H, Hussain I, Brust M, et al. Aligned two- and three-dimensional structures by directional freezing of polymers and nanoparticles. Nature Mater 2005; 4: 787-793.

15. Mansur HS and Costa HS. Nanostructured poly(vinyl alcohol)/bioactive glass and poly(vinyl alcohol)/chitosan/bioactive glass hybrid scaffolds for biomedical applications. Chem Eng $J$ 2008; 137: 72-83.

16. Shah AT, Batool M, Chaudhry AA, et al. Effect of calcium hydroxide on mechanical strength and biological properties of bioactive glass. $J$ Mech Behav Biomed Mater 2016; 61: 617-626.

17. Elzein T, Nasser-Eddine M, Delaite C, et al. FTIR study of polycaprolactone chain organization at interfaces. $J$ Colloid Interface Sci 2004; 273: 381-387.

18. Carta D, Knowles JC, Smith ME, et al. Synthesis and structural characterization of $\mathrm{P}_{2} \mathrm{O}_{5}-\mathrm{CaO}-\mathrm{Na}_{2} \mathrm{O}$ sol-gel materials. J Non Cryst Solids 2007; 353: 1141-1149.

19. Fowler B. Infrared studies of apatites. I. Vibrational assignments for calcium, strontium, and barium hydroxyapatites utilizing isotopic substitution. Inorg Chem 1974; 13: 194-207.

20. Shah A, Ain Q, Chaudhry A, et al. A study of the effect of precursors on physical and biological properties of mesoporous bioactive glass. $J$ Mater Sci 2015; 50: 1794-1804.

21. Ahsan MR and Mortuza MG. Infrared spectra of $x \mathrm{CaO}$ $(1-x-z) \mathrm{SiO}_{2} z \mathrm{P}_{2} \mathrm{O}_{5}$ glasses. J Non Cryst Solids 2005; 351: 2333-2340.

22. Trovati G, Sanches EA, Neto SC, et al. Characterization of polyurethane resins by FTIR, TGA, and XRD. J Appl Polym Sci 2010; 115: 263-268.

23. Shieh J-J and Chung TS. Effect of liquid-liquid demixing on the membrane morphology, gas permeation, thermal and mechanical properties of cellulose acetate hollow fibers. J Memb Sci 1998; 140: 67-79.

24. de Groot JH, Nijenhuis AJ, Bruin P, et al. Use of porous biodegradable polymer implants in meniscus reconstruction. 1) Preparation of porous biodegradable polyurethanes for the reconstruction of meniscus lesions. Colloid \& Polymer Sci 1990; 268: 1073-1081.

25. Aubert JH and Clough RL. Low-density, microcellular polystyrene foams. Polymer 1985; 26: 2047-2054.

26. Zhang M, Li XH, Gong YD, et al. Properties and biocompatibility of chitosan films modified by blending with PEG. Biomaterials 2002; 23: 2641-2648.

27. Khan A, Wong F, McKay I, et al. Structural, mechanical, and biocompatibility analyses of a novel dental restorative nanocomposite. J Appl Polym Sci 2013; 127: 439-447.

28. Mota J, Yu N, Caridade SG, et al. Chitosan/bioactive glass nanoparticle composite membranes for periodontal regeneration. Acta Biomaterialia 2012; 8: 4173-4180.

29. Rahim MI, Tavares A, Evertz F, et al. Phosphate conversion coating reduces the degradation rate and suppresses side effects of metallic magnesium implants in an animal model. J Biomed Mater Res 2017; 105: 1622-1635.

30. Aurer A and Jorgie-Srdjak K. Membranes for periodontal regeneration. Acta Stomatol Croat 2005; 39: 95-112. 